\title{
Abhandlung
}

\section{Vasile larmulschi* \\ „Feuerböcke“ der jüngeren Vorrömischen Eisenzeit im Milieu der Zarubincy-Kultur}

https://doi.org/10.1515/pz-2021-2009

Zusammenfassung: $\mathrm{Zu}$ den Objekten aus Ton, die in den Siedlungen der Zarubincy-Kultur von Belii Grud und Litvinovič 3 gefunden wurden, gehören sog. Feuerböcke. Bislang sind lediglich zwei solcher Exemplare bekannt. Die Analogien zu diesen Exemplaren sind im Milieu der Poienești-Lucașeuca-Kultur - Borosești, Lunca Ciurei sowie in Siedlungen des nördlichen Mitteleuropa, die in die Vorrömische Eisenzeit datiert werden - Bastrup, Hamburg-Volksdorf, Nowa Wieś, Ribe Amt usw. - zu verorten. Da die im nördlichen Mitteleuropa gefundenen Stücke älter sind, liegt der Ursprung der Feuerböcke wahrscheinlich im Norden.

Schlagwörter: Zarubincy-Kultur, jüngere Vorrömische Eisenzeit, Siedlungen, Feuerböcke, Migration.

\begin{abstract}
Among the objects made of clay found in the settlements of the Zarubincy culture some pieces are probably parts of hearth grids. So far, only two such specimens have been found in the settlements at Belîi Grud and Litvinovič 3 . The analogies to these specimens lay in the environment of the Poienesti-Lucaseuca culture - Borosesti, Lunca Ciurei - as well as in the settlements dated in the Late Pre-Roman Iron Age in Central-Northern Europe Bastrup, Hamburg Volksdorf, Nowa Wieś, Ribe Amt etc. As the objects found in northern Central Europe are older, Nordic origin of such hearth grids remains highly probable.
\end{abstract}

Keywords: Zarubincy culture, Late Pre-Roman Iron Age, settlements, hearth grids, migration.

Rezumat: Printre obiectele confecționate din lut, descoperite în așezările culturii Zarubincy, se numără și „cățeii de vatră“. Până în prezent se cunosc două astfel de exemplare. Acestea provin din așezările de la

\footnotetext{
*Corresponding Author: Vasile Iarmulschi, Freie Universität Berlin, Institut für Prähistorische Archäologie, FB Geschichts- u. Kulturwissenschaften, Fabeckstraße 23-25, 14195 Berlin, Germany, E-Mail: vasile.iarmulschi@gmail.com
}

Belîi Grud și Litvinovič 3. Cele mai apropiate analogii pentru aceste exemplare le cunoastem în mediul culturii Poienești-Lucașeuca - Borosești, Lunca Ciurei - cât și în siturile, datate în epoca preromană a fierului, din Europa Central-Nordică - Bastrup, Hamburg Volksdorf, Nowa Wieś, Ribe Amt etc. Întrucât descoperirile din mediul culturii Zarubincy se datează în epoca Latène-ului târziu, acceptăm ideea că aceste exemplare își au originea în Europa Central-Nordică.

Cuvinte-cheie: cultura Zarubincy, epoca preromană recentă a fierului, așezări, „căței de vatră“, migrație.

Gewidmet Professor Mircea Babeș

Wie aus bisherigen archäologischen Forschungen hervorgeht ${ }^{1}$, wirken sich auf das Gebiet des mittleren und oberen Dnjepr in der z wei te n Hälfte des 3. Jahrhunderts v. Chr. eine Reihe von sozialen, wirtschaftlichen und kulturellen Veränderungen aus. Als Ergebnis dieser Prozesse entsteht gegen Ende des 3. Jahrhunderts v. Chr. in jener Region die Zarubincy-Kultur ${ }^{2}$ (Abb. 1).

Obwohl diese archäologische Kultur nunmehr seit mehr als einem Jahrhundert Gegenstand wissenschaftlicher Forschungen ist, sind gewisse Aspekte der Zarubincy-Kultur, etwa ihre Entstehung und Chronologie, noch immer nicht vollständig geklärt. Dies erklärt die in der Fachliteratur geführten Diskussionen mit teils widersprüchlichen Thesen ${ }^{3}$.

Im Folgenden mochte ich verschiedene Aspekte der sog. - Herkunft, Chronologie, Funktionalität usw. - im Zusammenhang mit den sogenannten Feuerböcken, die

1 Максимов 1982; Еременко 1997; Пачкова 2006.

2 Zur Zarubincy-Kultur werden hauptsächlich drei Gruppen gerechnet: die Mitteldnjepr-, die Oberdnjepr- und die Polesien-Gruppe. Grundlage für die Abgrenzung dieser Gruppen waren vor allem Keramikformen sowie Tracht- und Bestattungsrituale (vgl. Еременко 1997; Пачкова 2006)

3 Максимов 1982; Каспарова 1993; Еременко 1997; Пачкова 2006; Дробушевский 2016; Woronjatov 2020. 


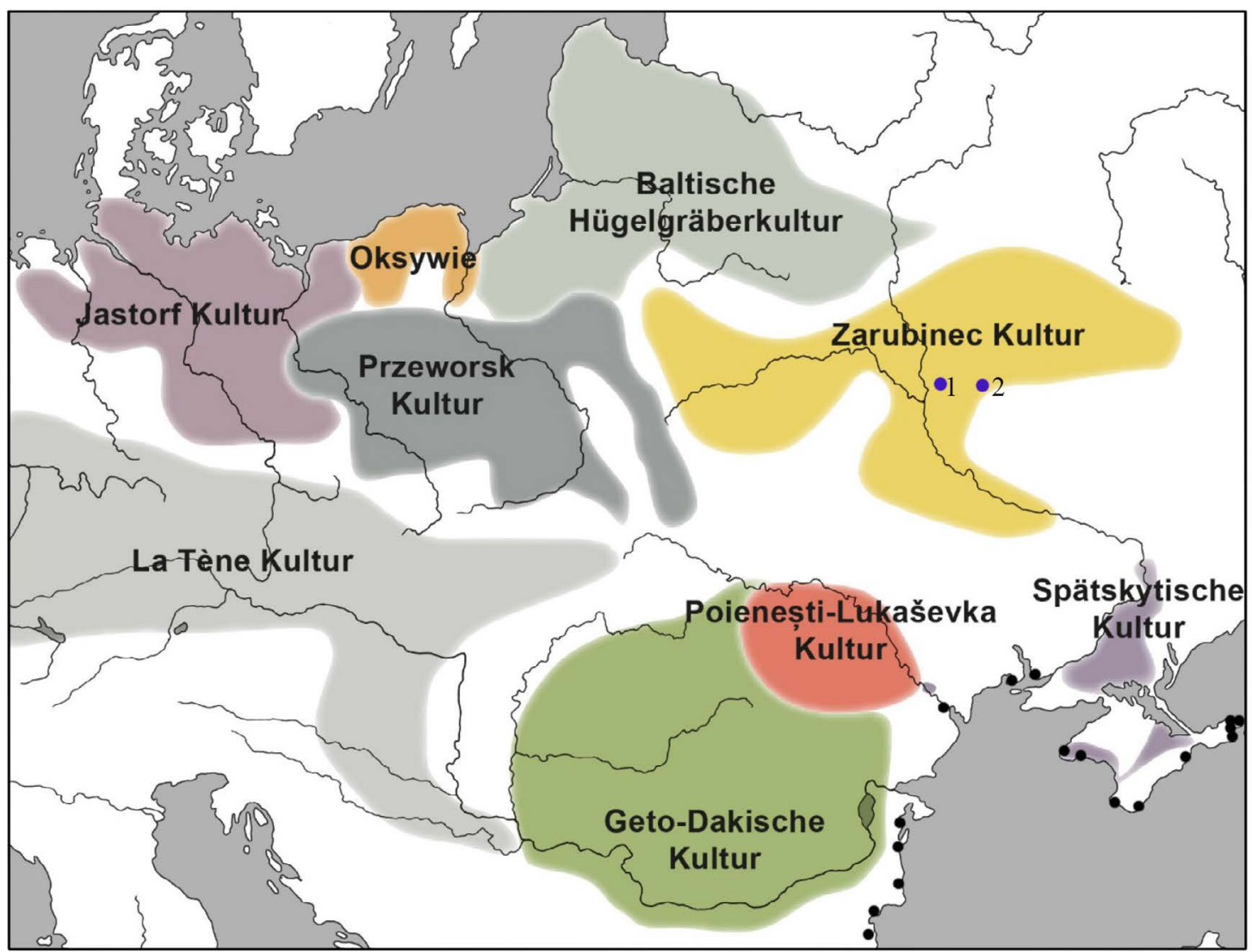

Abb. 1: „Archäologische Kulturen“ der jüngeren Vorrömischen Eisenzeit im nördlichen Mittel-und Südosteuropa. 1. Siedlung von Belîi Grud; 2. Siedlung von Litvinovič 3 (nach Hellström 2018; larmulschi 2020 mit Ergänzungen).

in den Siedlungen der Zarubincy-Kultur entdeckt wurden, vorstellen. Es sei zu erwähnen, dass bisher lediglich zwei solcher Objekte bekannt sind. Sie stammen aus den Siedlungen Belîi Grud ${ }^{4}$ und Litvinovič $3^{5}$.

\section{Kontext der Befunde und Beschreibung der Funde}

\section{Die Siedlung von Belyi Grud (reg. Černigova, Ukraine)}

Der Fundplatz befindet sich ca. 0,5 km südlich des Dorfes Starii Belous, auf der ersten Flussterrasse am linken Ufer des Belous. Bei den von A. V. Šekun durchgeführten archäologischen Ausgrabungen wurden zwei Häuser und mehrere Gruben mit Funden entdeckt, die der Zaru-

4 Шевченко 1997.

5 Обломский 2000.
bincy-Kultur zugeschrieben werden ${ }^{6}$. Es ist wichtig zu erwähnen, dass in der Nähe der Siedlung eine Nekropole entdeckt wurde, die teilweise übereinstimmend datiert. Nach Meinung des Autors sollte das Gräberfeld in den Zeitraum zwischen dem 1. Jahrhundert v. Chr. bis zum Beginn des 3. Jahrhunderts datiert werden ${ }^{7}$.

Im Befund Nr. 33, einer Grube, wurde neben mehreren Keramikfragmenten auch das Fragment eines Feuerbocks entdeckt. Das 7,5 cm $\times$ 4,9 cm große Stück in Pyramidenstumpfform zeichnet sich auf der Oberseite durch eine Plattform mit zwei gewölbten Seiten aus und ist an der Unterseite mit einem kleinen Loch im Boden abgeschlossen (Abb. 2,1).

\section{Siedlung Litvinovič 3 (Reg. Sumi, Ukraine)}

Der Fundplatz liegt auf der ersten Terrasse des Flusses Kleveni, etwa 0,35 km südlich der südöstlichen Grenze

6 Шевченко 1997, 122.

7 Ebd. 123; Шевченко 1997, 123. 


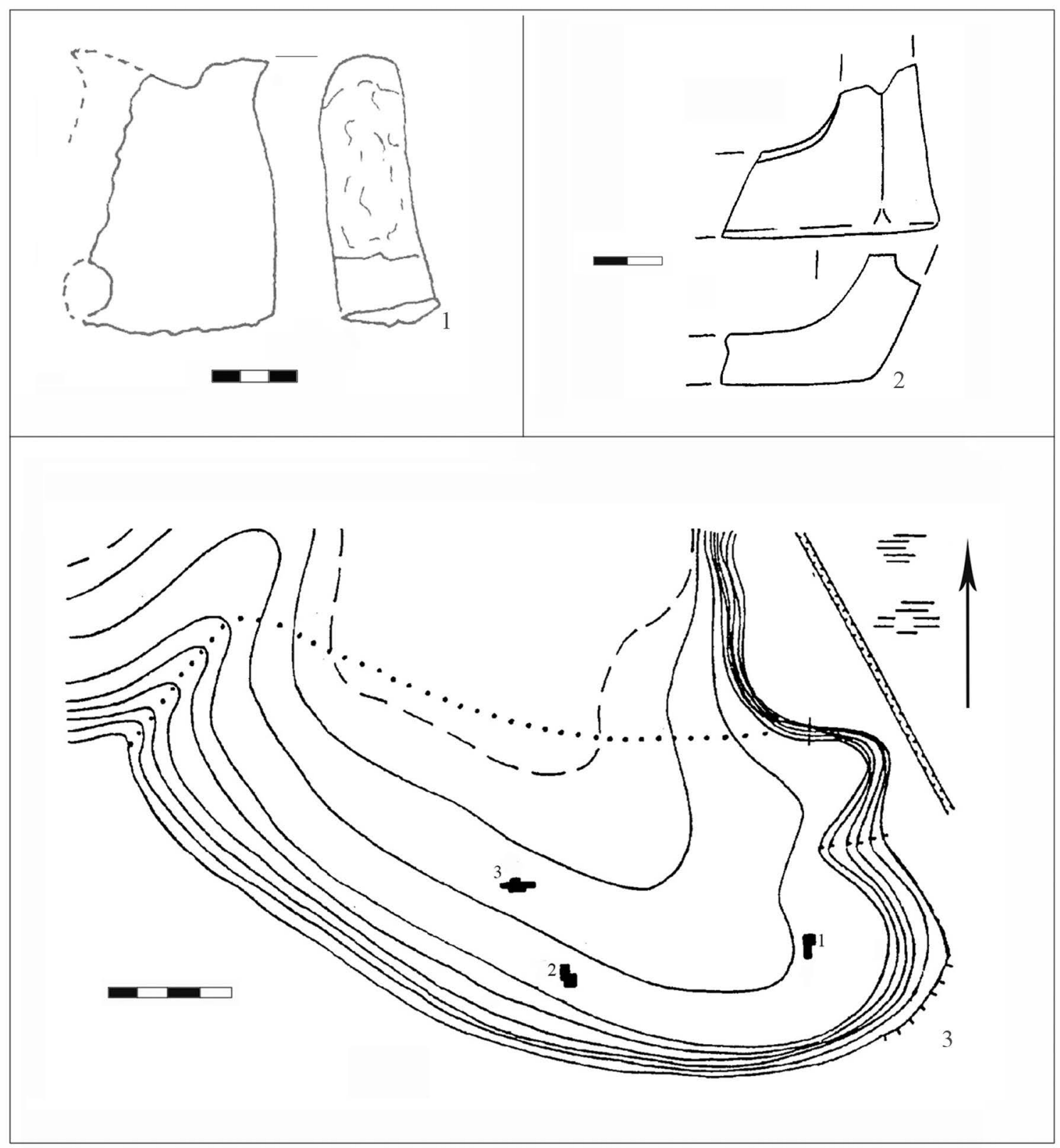

Abb. 2: Kontext der Befunde und Funde. 1. Der Feuerbock von Belîi Grud (nach Шевченко 1997);

2. Feuerbock von Litvinovič 3; 3. Plan der Ausgrabung bei Litvinovič 3 (nach Обломский 2000).

des Dorfes. Die Siedlung nimmt eine Fläche von ca. $600 \mathrm{~m} \times 300 \mathrm{~m}$ ein. Bei den von A. M. Oblomskii durchgeführten archäologischen Ausgrabungen wurde eine Fläche von $432 \mathrm{~m}^{2}$ freigelegt (Abb. 2,3), auf der ein Haus und 15 Gruben, die der Zarubincy-Kultur zugeschrieben werden $^{8}$, ausgegraben wurden ${ }^{9}$. In der Kulturschicht

$8 \mathrm{Zu}$ erwähnen ist, dass vom Fundplatz auch bronzezeitliche, spathallstattzeitliche und mittelalterliche Siedlungsspuren stammen. 9 Обломский 2000, 141. des Fundplatzes konnte neben Keramikfragmenten und Werkzeugen auch das Fragment eines Feuerbocks entdeckt werden. Das 5,5 cm $\times 6,4 \mathrm{~cm}$ große Stück hatte wahrscheinlich die Form einer stumpfen Pyramide, mit einer Plattform mit zwei gewölbten Bereichen an der Oberseite. Der untere Teil des Stücks hatte an allen vier Seiten „Fenster“ (Abb. 2,2). 


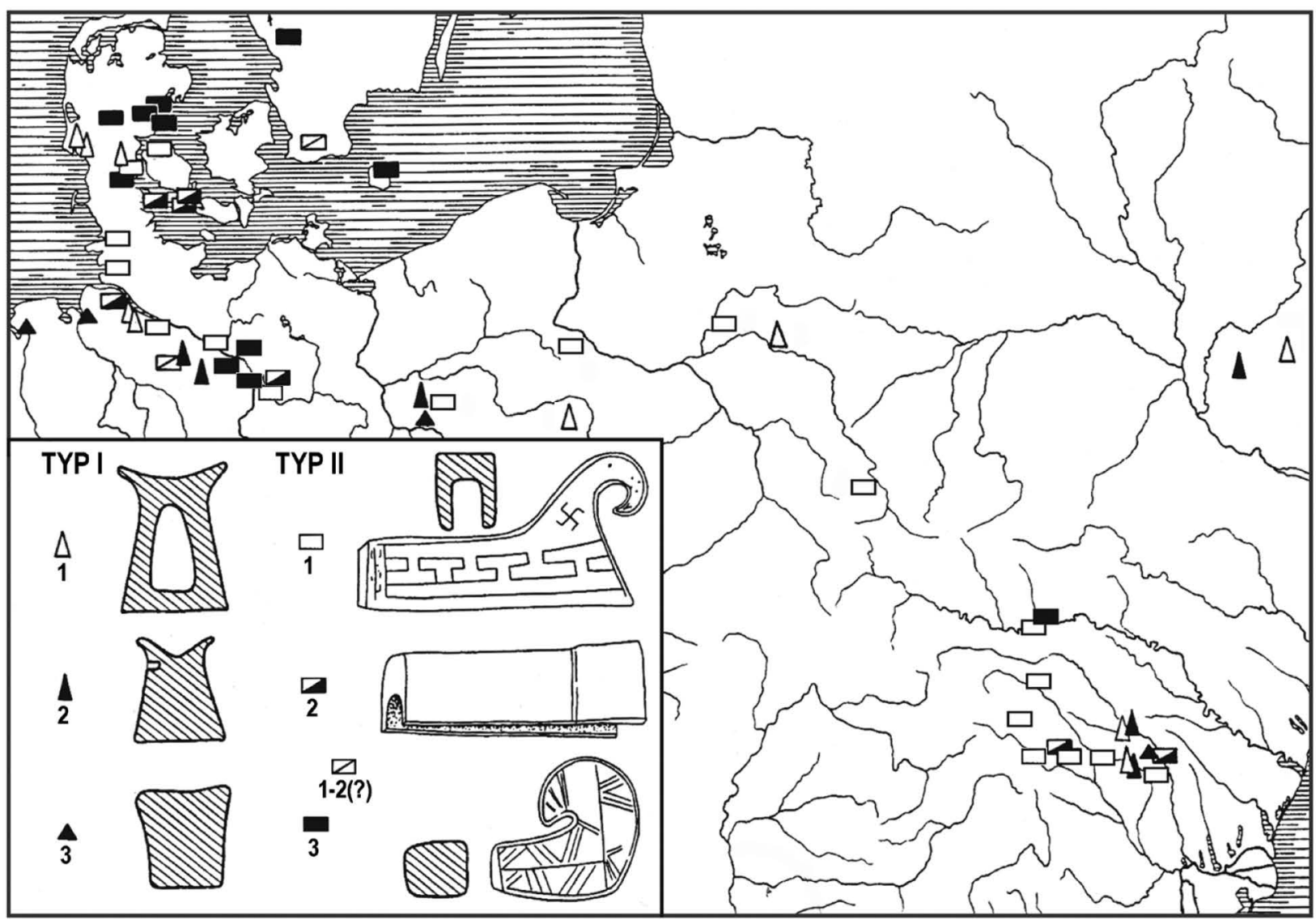

Abb. 3: Verbreitung der latènezeitlichen Feuerböcke in Mittel- und Osteuropa (nach Babeș 1993; Michałowski/Teska 2012; Michałowski et al. 2018; Babeș/larmulschi 2020 mit Ergänzungen).

\section{Typologie, Verbreitung und Herkunft}

In einer ausgezeichneten Studie, die solchen Exemplaren gewidmet ist, hatte 1970/71 der Archäologe M. Babeș ${ }^{10}$ zwei Hauptformen von Feuerböcken eingegrenzt:

1. den Borosești-Typ - dieser zeichnet sich durch seine Pyramidenstumpfform, oben mit erhöhten Schmalseiten endend, aus;

2. den Ghelăiești-Typ, welcher durch die langgestreckte Form des Körpers, der in einer Volute endet, gekennzeichnet ist (Abb. 3).

Die im Milieu der Zarubincy-Kultur gefundenen Exemplare können nach der Klassifizierung von M. Babeș ${ }^{11}$ dem Typ 1 (Borosești) zugeordnet werden. Dem Autor sind ähnliche Exemplare sowohl aus der Poienești-Lucașeuca-Kultur

10 Babeș/Mihăilescu-Bîrliba 1970/1971.

$11 \mathrm{Zu}$ vermerken ist, dass nach Babeș/Mihăilescu-Birliba 1970/1971 noch drei Varianten zum Typ rechnen. Das Stück aus Litvinovič 3 entspricht der Variante 1 und das aus Belîi Grud der Variante 2. als auch aus Siedlungen im nördlichen Mitteleuropa bekannt (Abb. 3). Die besten Analogien zum Exemplar von Litvinovič 3 stammen aus den Siedlungen von Borosești ${ }^{12}$ (Abb. 4,1-2) und Lunca Ciurei ${ }^{13}$.

Ähnliche Feuerböcke sind im heutigen Dänemark bekannt. Unter den Funden sind die Exemplare aus Bastrup, Ribe $\mathrm{Amt}^{14}$ (Abb. 5,1), Vognsbøl und Sjælborg ${ }^{15}$ (Abb. 5,2-3) zu erwähnen. Analogien zum Stück aus Litvinovič 3 finden sich auch in Norddeutschland, ein ähnliches Exemplar wurde in der Siedlung HamburgVolksdorf gefunden ${ }^{16}$. Kürzlich wurde ein dem Typ I.1 zugeschriebenes Stück auch in der Przeworsk-Typ-Siedlung von Daniszew stan. 18 nachgewiesen ${ }^{17}$ (Abb. 6,1).

12 Babeș 1993, Taf. 16,1; Babeș/Iarmulschi 2020, Pl. 1,1; 3,9. 13 Teodor 1987, 91 Abb. 29,8.11; Babeș 1993, Taf. 30,1-2.

14 Müller 1907, 132 Abb. 39.

15 Thomsen 1959, 24 Abb. 11-12.

16 Bücke 2007, 135-136 Abb. 61.

17 Michalowski et al. 2018. 


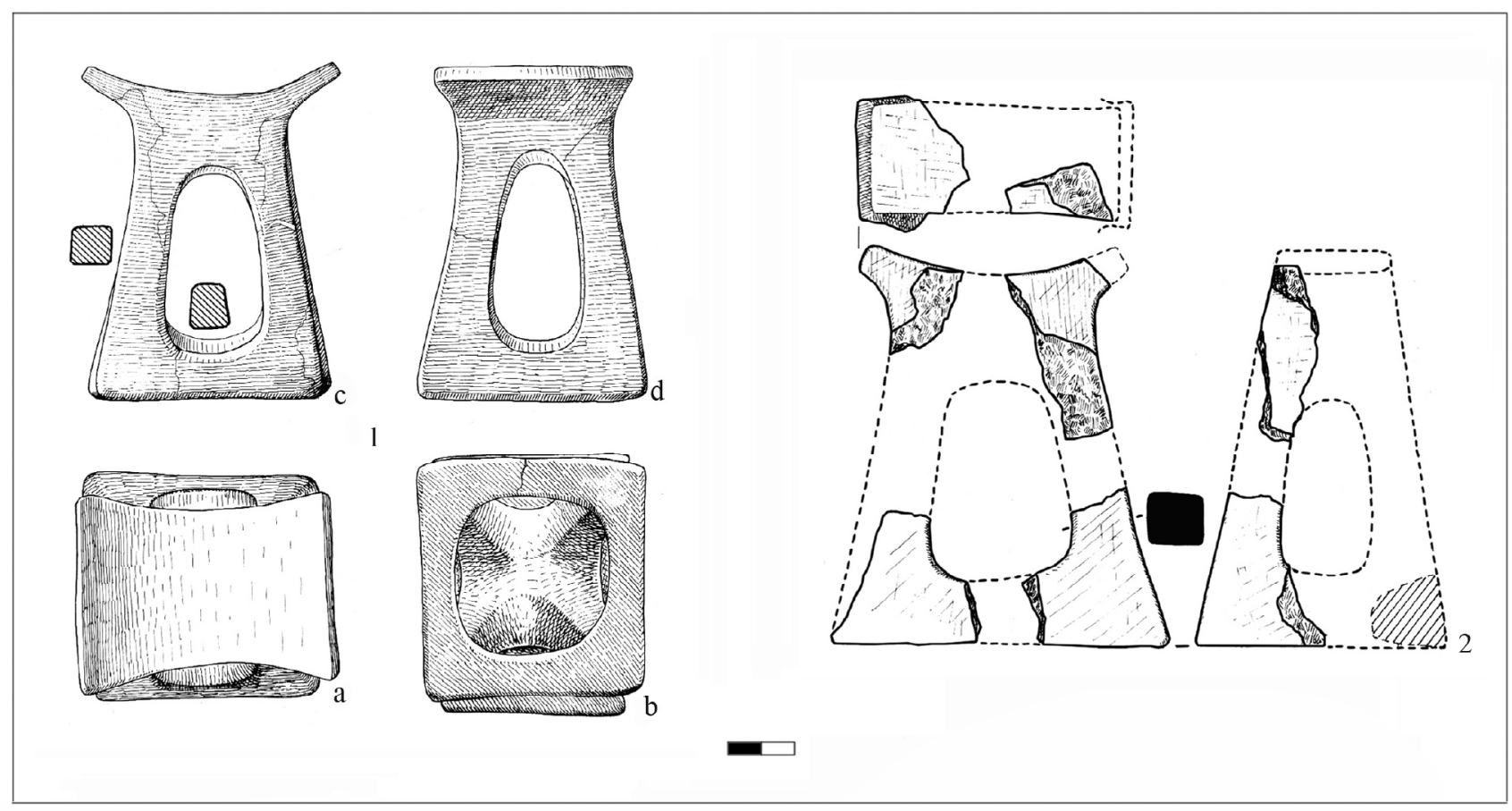

Abb. 4: Feuerböcke des Typs I.1 aus der Siedlung von Borosești (nach Babeș 1993; Babeș/larmulschi 2020).

Für das in Belîi Grud gefundene Exemplar tauchen auch Analogien in der Poienești-Lucașeuca-Kultur sowie im nördlichen Mitteleuropa auf. So wurden in der ostkarpatischen Waldsteppe vergleichbare Feuerböcke in den Siedlungen von Borosești ${ }^{18}$ (Abb. 6,2) und Lunca Ciurei ${ }^{19}$ gefunden (Abb. 6,3).

Parallelen für das Stück aus Belîi Grud sind von der Halbinsel Jütland ${ }^{20}$ (Abb. 6,4) und den spätvorrömischen eisenzeitlichen Siedlungen bei Lehmke und Vahlfitz in Niedersachsen bekannt ${ }^{21}$. Solche Objekte wurden auch in der Siedlung der Przeworsk-Kultur von Nowa -Wieś gefun$\operatorname{den}^{22}$ (Abb. 7,1-2). Ein weiteres Stück wurde in der Przeworsk-Nekropole in Strzyżowie entdeckt ${ }^{23}$ (Abb. 7,3), ein Fragment eines Feuerbock Typ I.1. nach Babeș stammt aus einer Siedlung der Tyniec-Gruppe bei Krakow-Wyciąż ${ }^{24}$ (Abb. 7,4).

Da die im nördlichen Mitteleuropa, speziell in Dänemark, gefundenen Stücke älter sind, bleibt der nordische

18 Babeș 1993, Taf. 18,15; Babeș/Iarmulschi 2020, Pl. 6,11. 19 Teodor 1987, 91; Babeș 1993, 77 Taf. 31,1; Iarmulschi 2020, 76. 20 Martens 2017, pl. 14,2.

21 Sprockhoff 1940, 248 Abb. 3.

22 Dzieduszycki/Makiewicz/Sobucki 1998, 159 ryc. 29,1; 30,3.4.6; Michałowski 2010, 177 Abb. 10; Michałowski/Teska 2012, 327.

23 Dąbrowska 1978, 78 Tab. 1,1.17; Dąbrowska 1994, 73.

24 Woźniak/Poleska 1999, 384-385 ryc. 3,p.
Ursprung der Feuerböcke vom Borosești-Typ sehr wahrscheinlich $^{25}$.

\section{Chronologie}

Zur Chronologie der im Gebiet der Zarubincy-Kultur entdeckten Feuerböcke liegen keine relevanten Untersuchungen vor. Wie bereits erwähnt, wurde das Exemplar aus Litvinovič 3 in der Kulturschicht der Siedlung gefunden. Anhand von Importmaterial (Abb. 8,2-6) und einer Fibel der Variante Kostrzewski N (Abb. 8,1), konnte der Fundplatz zwischen das Ende des 3. Jahrhunderts v. Chr. und den ersten Jahrzehnten des 1 . Jahrhunderts n. Chr. datiert werden ${ }^{26}$.

Das Stück von Belîi Grud wurde in einer Grube mit Keramik, die für die klassische Phase der Zarubincy-Kultur charakteristisch ist, gefunden ${ }^{27}$. Der Ausgräber von Belii Grud nahm an, dass die Siedlung zum Beginn des 1. Jahrhunderts v. Chr. gegründet wurde ${ }^{28}$. Solange die Ergebnisse der Ausgrabungen jedoch nur teilweise veröffentlicht sind, kann ich diesen Ansatz nicht teilen.

25 Babeș 1993, 78; Babeș/Iarmulschi 2020, 109.

26 Обломский 2000, 153.

27 Шевченко 1997, 123.

28 Ebd. 122. 


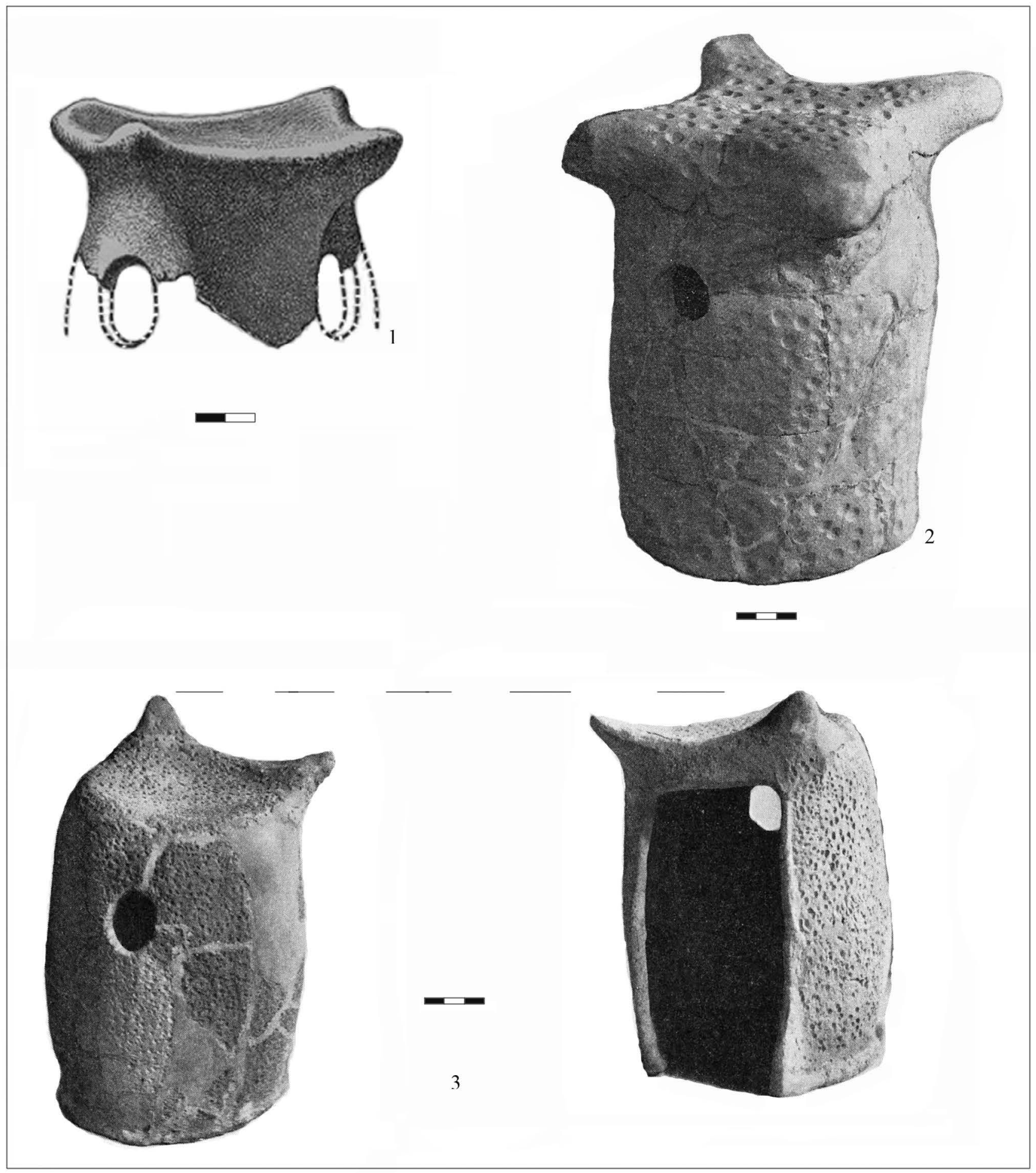

Abb. 5: Feuerböcke des Typs I.1 aus dem nördlichen Mitteleuropa. 1. Feuerbock aus der Siedlung Bastrup (nach Müller 1907); 2-3. Exemplare aus den Siedlungen Vognsbøl und Sjælborg (nach Thomsen 1959). 


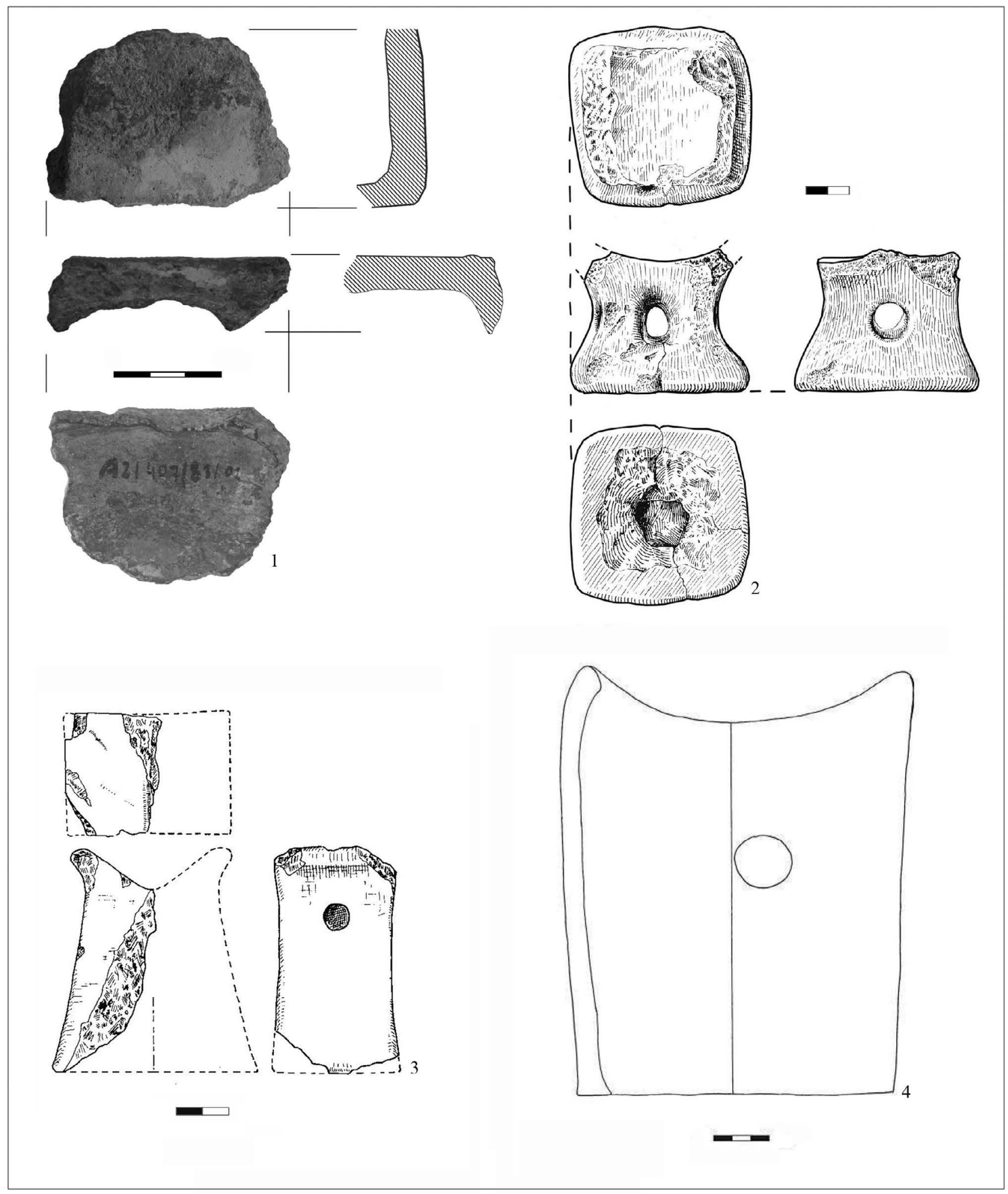

Abb. 6: Feuerböcke aus Mittel- und Südosteuropa. 1. Exemplar von Daniszew, stan. 18 (nach Michałowski et al. 2018); 2. Feuerbock aus der Siedlung von Borosești (nach Babeș 1993; Babeș/larmulschi 2020); 3. Exemplar aus der Siedlung von Lunca Ciurei (nach Babeș 1993); 4. Feuerbock aus der Siedlung von Borremose (nach Martens 2017). 


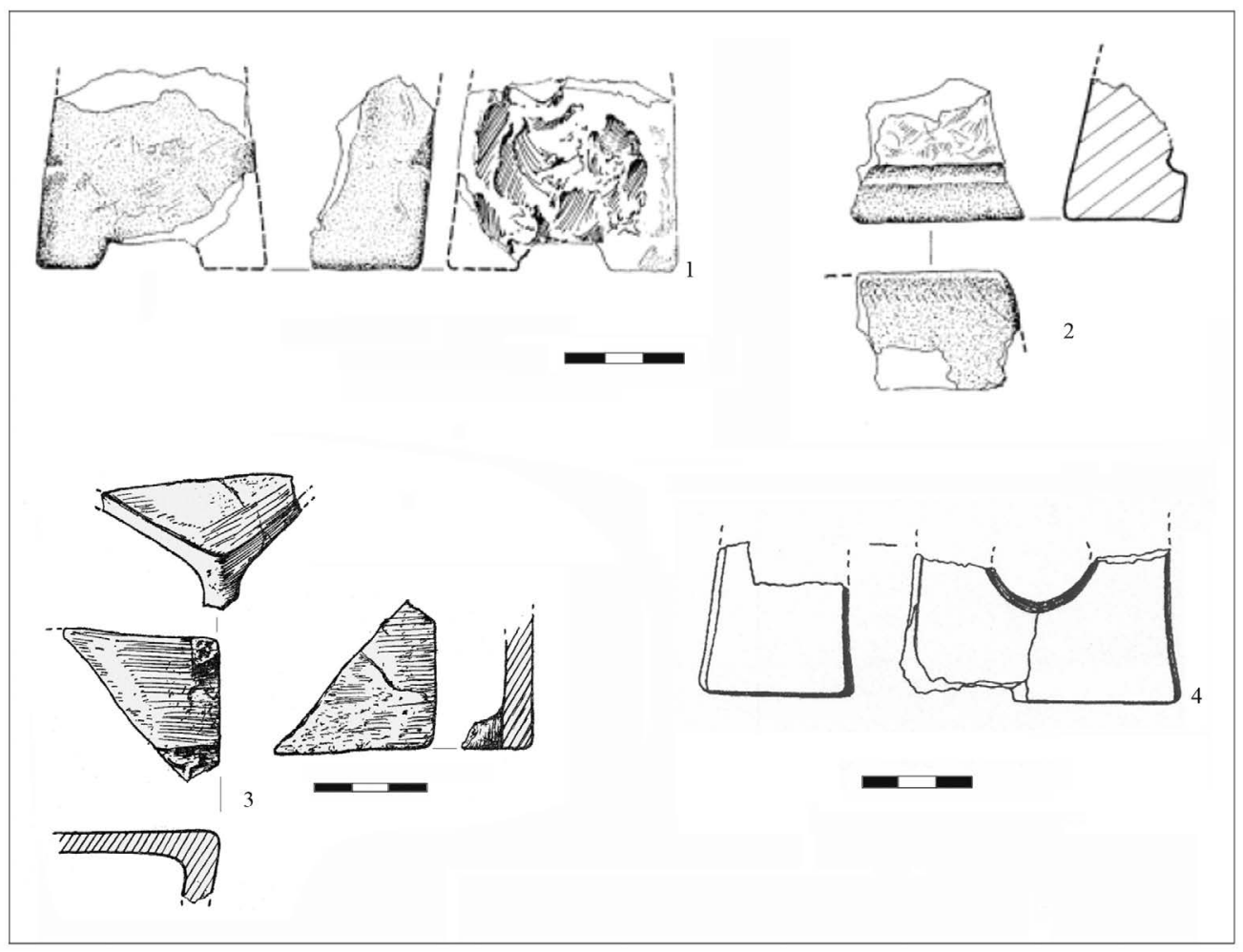

Abb. 7: Feuerböcke aus Mitteleuropa. 1-2. Exemplare aus der Siedlung von Nowa Wieś (nach Michałowski 2010); 3. Feuerbock aus dem Gräberfeld von Strzyżowie (nach Dąbrowska); 4. Exemplar von Krakow-Wyciąż (nach Woźniak/Poleska 1999).

Chronologisch ableiten lässt sich also, dass die Feuerböcke der Zarubincy-Kultur grob in den Zeitraum zwischen das Ende des 3. Jahrhunderts v. Chr. und den Anfang des 1. Jahrhunderts n. Chr. datieren. Anzunehmen ist jedoch, dass mittels Extrapolation der Funddatierungen aus der Poienești-Lucașeuca-Kultur die Chronologie dieser Stücke eingegrenzt werden kann. So habe ich bei der Diskussion des Problems der chronologischen Einordnung und Periodisierung der Poienești-Lucașeuca-Kultur vorgeschlagen, dass die Feuerböcke (Typ 1 nach Babeș) der zweiten Entwicklungsphase der Kultur zugerechnet werden sollten ${ }^{29}$, was einem Zeitraum zwischen dem zweiten und dem letzten Viertel des 2. Jahrhunderts v. Chr entspricht. Entsprechend gehe ich davon aus, dass auch die Feuerböcke aus Belîi Grud und Litvinovič 3 in diesen Zeitraum datieren.

\section{Funktionalität}

Bezüglich der Funktionalität der Feuerböcke ist festzustellen, dass in der Literatur verschiedene Meinungen vorherrschen. So sind einige Forscher der Ansicht, dass solche Stücke konstitutive Elemente von „Öfen“ innerhalb von Häusern waren ${ }^{30}$. Andere wiederum vertreten die Meinung, dass diese Fundstücke als Stützen für Gefäße dienten, die in der Nähe der Feuerstellen aufgestellt waren, wiederum Dritte nahmen an, dass sie als „Heizplatten“ (auch ,Wärmflaschen') verwendet wurden ${ }^{31}$. Gleichzeitig ist nicht ausgeschlossen, dass solche Funde eine kultische Konnotation hatten ${ }^{32}$.

Nach der Art und Weise, wie der obere Bereich der in den Fundstellen der Zarubincy-Kultur gefundenen Feuerböcke - eine Plattform mit zwei gewölbten Seiten - angeordnet ist, gehe ich davon aus, dass diese Feuerböcke sehr wahrscheinlich als Untersteller für Töpfe oder als „Heizplatten" verwendet wurden.

30 Schirren 1992-1993, 37. 31 Bücke 2007, 163-166; Nüsse 2008, 53; Moser/Biermann 2018, 137-138.

32 Steuer 1973, 216; Schneeweiß 2013, 186.
29 Iarmulschi 2013, 47 Abb. 18; 2016, 489 Abb. 16; Babeș/Iarmulschi 2020, 138. 


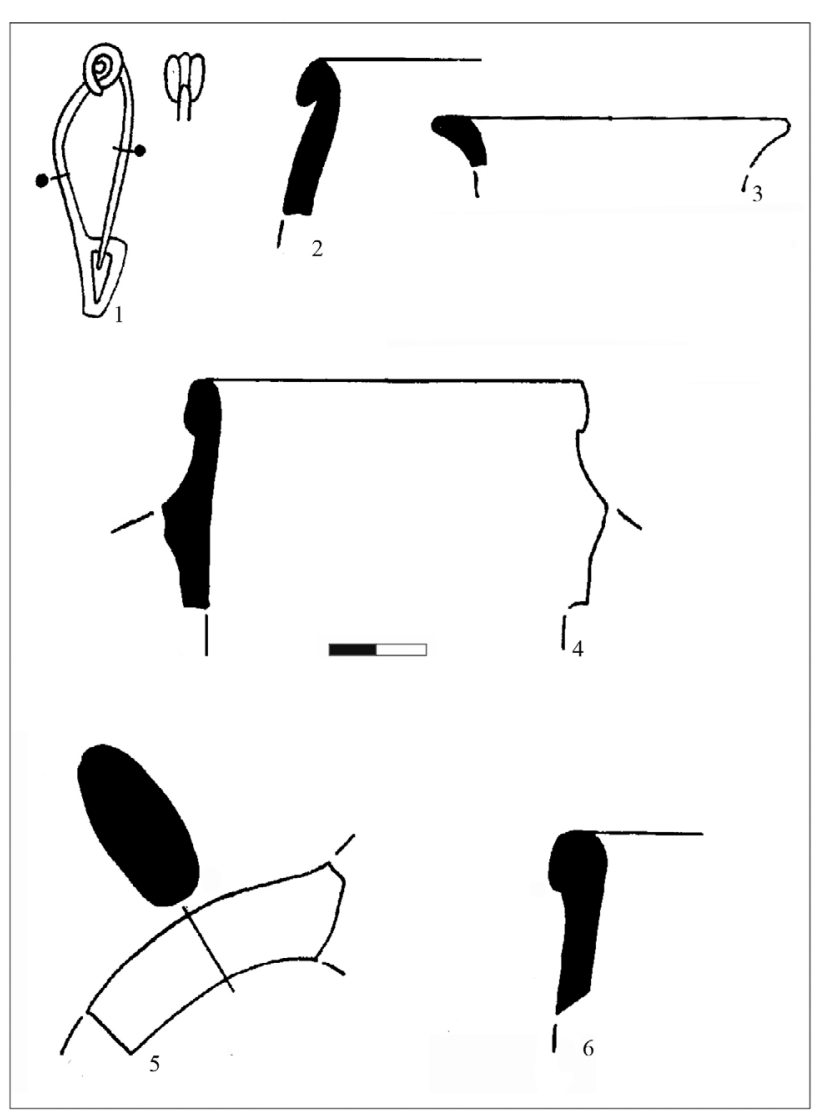

Abb. 8: Fibel und „griechische Importe“ aus der Siedlung von Litvinovič 3 (nach Обломский 2000).

\section{Fazit}

Aus dem Milieu der Zarubincy-Kultur sind uns bislang zwei fragmentarisch erhaltene Exemplare bekannt, die als Feuerböcke bezeichnet werden können. Solche Exemplare, die wahrscheinlich als Untersteller für Töpfe oder als „Heizplatten“ verwendet wurden, sollten in das 2. Jahrhundert v. Chr. datiert werden.

Die Feuerböcke, die in der Zarubincy-Kultur gefunden wurden, dokumentieren meiner Meinung nach die Existenz von Beziehungen zwischen dem nördlichen Mitteleuropa und dem mittleren Dnejpr-Gebiet in der jüngeren Vorrömischen Eisenzeit. Wie im Fall der PoieneștiLucașeuca-Kultur haben wohl Bevölkerungsgruppen aus dem nördlichen Mitteleuropa beim Entstehungsprozess der Zarubincy-Kultur eine Rolle gespielt. Tatsächlich wurde der Fund dieser Exemplare im heutigen Polen von vielen Wissenschaftlern als Beweis für eine Migration von der Halbinsel Jütland und Norddeutschland in die nordwestlichen Regionen des Schwarzen Meeres interpretiert ${ }^{33}$.

So vervollständigen die im Gebiet der Zarubincy-Kultur entdeckten Feuerböcke mit neuen Daten verschiedene Aspekte im Zusammenhang mit der Migration und Besiedlung der Regionen am mittleren und oberen Dnjepr in der jüngeren Vorrömischen Eisenzeit durch aus dem nördlichen Mitteleuropa stammende Populationen. Ich bin davon überzeugt, dass sowohl zukünftige Entdeckungen als auch die Auswertung der bisherigen Funde mehr Licht und Klarheit in die Lösung dieser historisch-archäologischen Fragen bringen werden.

\section{Literaturverzeichnis}

Babeș 1993: M. Babeș, Die Poienești-Lukaševka-Kultur. Ein Beitrag zur Kulturgeschichte in Raum östlich der Karpaten in den letzten Jahrhunderten vor Christi Geburt. Saarbrücker Beiträge zur Altertumskunde 30 (Bonn 1993).

-/Mihăilescu-Bârliba 1970/1971: -/V. Mihăilescu-Bârliba, Germanische „Feuerböcke“ aus der Moldau. Berichte der Römisch-Germanische Kommission 51-52, 1970-1971, 176-196.

-/larmulschi 2020: -/V. Iarmulschi, Așezarea și necropola de tip Poienești-Lucașeuca de la Borosești. Tyragetia XXXII (Chișinău 2020).

Brandt 2001: J. Brandt, Jastorf und Latène. Kultureller Austausch und seine Auswirkungen auf soziopolitische Entwicklungen in der vorrömischen Eisenzeit. Internationale Archäologie 66 (Rahden/Westf. 2001).

Bücke 2007: S. Bücke, Siedlungsfunde der jüngeren vorrömischen Eisenzeit aus Hamburg-Volksdorf. Internationale Archäologie 101 (Rahden/Westf. 2007).

Dąbrowska 1978: T. Dąbrowska, Cmentarzysko kultury przeworskiej i wielbarskiej na stanowisku „Kozarówka“ w Drohiczynie, woj. Białotosckie. Wiadamości Archeologiczne 43, 1978, 62-81.

- 1994: -, Wpływy jastorfskie na kulturę przeworska w młodszym okresie przedrzymskim. In: H. Rayss (Red.), Kultura przeworska (Lublin 1994) 71-88.

Dzieduczycki/Makiewicz/Sobucki 1998: W. Dzieduczycki/T. Makiewicz/A. Sobucki, Nowa Wieś, st. 1 I 2, gmina Bledzew, GAZ 43/43a, AZP 51-12:7 i 55. In: R. Mazurowski (Ed.), Archeologiczne badania ratownicze wzdłuż trasy gazociągu tranzytowego. Tom I. Ziemia lubuska (Poznań 1998) 117-181.

Hellström 2018: K. Hellström, Fibeln und Fibeltracht der Sarmatischen Zeit im Nordschwarzmeergebiet (2. Jh. v. Chr 3. Jh. n. Chr.). Archäologie in Eurasien 39 (Bonn 2018).

larmulschi 2013: V. Iarmulschi, Unele considerații privind cronologia culturii Poienești-Lucașeuca. Tyragetia s. n. VII/1, 2013, 29-52.

- 2016: -, A few consideration regarding the chronology of the Poienești-Lucașeuca culture. Praehistorische Zeitschrift 91/2, 2016, 471-494.

33 Dąbrowska 1994, 73; Michałowki/Teska, 2012,341; Michałowki et al. 2018, 498-499. 
- 2020: -, The Settlements of the Poieneşti-Lucaşeuca Culture. Archaeological study of settlements in the Carpathian-Dniester region from the end of the $3^{\text {rd }}$ to the $1^{\text {st }}$ century $B C$. Berliner Archäologische Forschungen 19 (Rahden/Westf. 2020).

Martens 1996: J. Martens, Die vorrömische Eisenzeit in Südskandinavien, Probleme und Perspektiven. Praehistorische Zeitschrift 71, 1996, 217-243.

- 2017: -, Settlement Pottery of the Kraghede Group. In: M. Meyer/ P. Łuckziewicz/B. Rauchfuß (Hrsg.), Eisenzeitliche Siedlungskeramik der Przeworsk-Kultur. Berlin Studies of the Ancient Word 50 (Berlin 2017) 153-198.

- 2021: -, Chronologie und Klima. In: E. Sava/V. Iarmulschi/ A. Zanoci, M. Băț/O. Munteanu (Hrsg.), Die Chronologie der vorrömischen Eisen- und frühen Kaiserzeit in Mittel- und Südosteuropa: Probleme und Perspektiven (Chișinău 2020; Berlin 2021) 63-74.

Michałowski 2010: A. Michałowski, Die Siedlungen der Jastorf-Kultur in Großpolen. In: M. Meyer (Hrsg.), Haus-Gehöft-WeilerDorf. Siedlungen der Vorrömischen Eisenzeit in nördlichen Mitteleuropa (Berlin 2010) 169-198.

-/Teska 2012: -/M. Teska, Nowe odkrycia figurek Feuerböcke z terenów ziem polskich. Folia Praehitorica Posnaniensia XVI, 2012, 331-344.

- et. al. 2018:-/A. Sikorski/M. Teska/P. Kaczmarska, Wilk ogniowy z odciskiem tkaniny płóciennej z miejscowości Daniszew, gm. Kościelec, pow. Kolski, stan. 18. In: B. NiezbitowskaWiśniewska/P. Łuczkiewicz/S. Sadowski/M. Stasiak/M. Erdrich (Red.), Studia Barbarica. For Professor A. Kokowski on his 65th birthday (t. II) (Lublin 2018), 492-501.

Moser/Biermann 2018: A. Moser/E. Biermann, „Feuerböcke“ der vorrömischen Eisenzeit - eine vernachlässigte Artefaktgruppe. Funde aus Karstädt 12 und Garlin 10, Lkr. Prignitz. Veröffentlichungen zur brandenburgischen Landesarchäologie 48, 2018, 127-139.

Müller 1907: S. Müller, Saakaldte Ildbukke Altre af Ler. Aarhøger 22, 1907, 127-136.

Nüsse 2008: H.-J. Nüsse, Untersuchungen zur Besiedlung des Hannoverschen Wendlands von der jüngeren vorrömischen Eisen- bis zur Völkerwanderungszeit. Neue Ausgrabungen und Forschungen in Niedersachsen 26 (Neumünster 2008).

Schirren 1992-1993: C. M. Schirren Ziegelförmige Tonklötze in der Eisenzeit. Ein Neufund aus Juliusburg, Kr. Herzogtum Lauenburg. Offa 49-50, 1992-1993, 33-39.
Schneeweiß 2013: J. Schneeweiß, Hirschkult bei den Germanen, Die Deponierung von Hirschgeweih und Feuerbock aus Vietze an der Elbe. In I. Heske/H.-J. Nüsse/J. Schneeweiß (Hrsg.), Landschaft, Besiedlung und Siedlung. Archäologischen Studien im nordeuropäische Kontext. Festschrift für K.-H. Willroth zu seinem 65. Geburtstag (Neumünster/Hamburg 2013) 177-190.

Sprockhoff 1940: E. Sprockhoff, Eine Elbgermanische Siedlung der Spätlatènezeit von Lehmke, Lkr. Uelzen. Germania 24, 1940, 243-251.

Steuer 1973: H. Steuer, Germanische „Feuerböcke“ aus dem Hannoverschen Wendland. Archäologisches Korrespondenzblatt 3, 1973, 213-217.

Teodor 1987: S. Teodor, Cercetările arheologice de la Ciurea. ArhMold XI, 1987, 65-102.

Thomsen 1959: N. Thomsen, Hus og kælder I romesk jernalder. Kuml 1959, 13-27.

Woronjatov 2020: S. Woronjatov. Die Bastarnen und die SarubintzyKultur. In: Ausstelungskatalog - Eisenzeit. Europa ohne Grenzen. 1 Jahrtausend v. Chr. (Sankt Petersburg 2020).

Woźniak/Poleska 1999: Z. Woźniak/P. Poleska, Zabytki tipu jastorfskiego z zachodniej Malopolski. In: J. Andrzejowski (Red.), COMHLAN (Warszawa 1999) 379-394.

Дробушевский 2016: Зарубинецкая культура Белорусского Поднепровья. In: ред. А. А. Коваленя/В. В. Данилович (отв.), Славяне на территории Белоруссии в догосударственный период.

Еременко 1997: В. Е Еременко, «Кельтская вуаль» и зарубинецкая культура (СПб. 1997).

Каспарова 1993: К. В. Каспарова, О времени возникновения зарубинецкой культуры, Археологические Вести 2, 1993 , 169-190.

Максимов 1982: Е. В. Максимов, Зарубинецкая культура на территории УРСС (Киев 1982).

Пачкова 2006: С. П. Пачкова, Зарубинецкая культура и латенизированные культуры Европы (Киев 2006).

Обломский 2000: А. М. Обломский, Материалы зарубинецкого периода на поселений Литвиновичи-3 в среднем Посейме. Stratum plus 4, 2000, 141-155.

Шевченко 1997: Ю. Ю. Шевченко, Высокий (Белый) Груд на рубеже эр. Stratum plus, 1997, 121-137. 